\title{
BENDING, BUCKLING AND FREE VIBRATION OF A BEAM WITH UNSYMMETRICALLY VARYING MECHANICAL PROPERTIES
}

\author{
KRZYSZTOF MagnuCKI, JeRZY LEWIŃSKI \\ Institute of Rail Vehicles TABOR, Poznań, Poland \\ Ewa MagnuCKa-BlandZi \\ Poznan University of Technology, Institute of Mathematics, Poznań, Poland \\ e-mail: ewa.magnucka-blandzi@put.poznan.pl \\ PIOTR KĘDZIA \\ Poznan University of Technology, Institute of Applied Mechanics, Poznań, Poland
}

\begin{abstract}
The subject of the paper is a beam with unsymmetrically varying mechanical properties in the depth direction. The nonlinear hypothesis of plane cross section deformation is assumed. Based on Hamilton's principle, two differential equations of motion are obtained. The system of equations is analytically solved with a view to analyse the bending, buckling and free vibration problems of the beam. Moreover, the FEM model of the beam is developed and deflections, critical axial forces and natural frequencies of the beam are calculated. The results of these two methods are compared.
\end{abstract}

Keywords: FGM beam, mathematical modelling, numerical FEM calculations

\section{Introduction}

Elements with varying mechanical properties are applied in modern constructions. Kubiak (2005) presented dynamic buckling problems of thin-walled composite plates with varying width-wise material properties. Zhang et al. (2006) presented free vibration analysis of rectangular composite laminated plates. Zenkour (2006) analysed bending problems of rectangular functionally graded plates under a transverse uniform load. Birman and Byrd (2007) presented a review of the papers published since 2000 related to the modelling and analysis of functionally graded materials and structures. Kapuria et al. (2008) described the theoretical model of bending and free vibration of layered functionally graded beams and its experimental validation. Debowski et al. (2010) studied the dynamic stability problem of a metal foam rectangular plate under compression in the middle plane. Magnucka-Blandzi (2011) presented bending and dynamic stability results of studies of the sandwich beam with a metal foam core. Kubiak (2011) described an estimation problem of dynamic buckling for composite columns with open cross-sections. Thai and Vo $(2012,2013)$ presented bending, buckling, and vibration of functionally graded beams and plates with the use of nonlinear shear deformation theories. Mahi et al. (2015) presented bending and free vibration analysis of isotropic, functionally graded sandwich and laminated composite plates with the use of a new hyperbolic deformation theory. Kolakowski and Mania (2015) presented the dynamic response of thin functionally graded plates with a static unsymmetrical stable postbuckling path. Chen et al. (2015, 2016a,b) analysed static bending, elastic buckling and free vibrations problems of shear deformable functionally graded porous beams and sandwich beams with a functionally graded porous core. Jun et al. (2016) studied the free vibration problem of axially loaded laminated composite beams using a unified higher-order shear deformation theory and a dynamic stiffness method. Mojahedin et al. (2016) presented 
the buckling problem of functionally graded circular plates with symmetrically and unsymmetrically varying mechanical properties based on a higher order shear deformation theory. Li and $\mathrm{Hu}$ (2016) analysed nonlinear bending and free vibration problems of nonlocal strain gradient beams made of a functionally graded material. Feyzi and Khorshidvand (2017) presented the axisymmetric post-buckling behaviour problem of saturated porous circular plates. Song et al. (2017) described vibration problems of functionally graded polymer composite plates reinforced with graphene nanoplatelets. Smyczynski and Magnucka-Blandzi (2018) presented a comparison of the study results of three-point bending of a sandwich beam with two binding layers with the use of two nonlinear hypotheses. Sayyad and Ghugal (2017) presented an extensive review of the papers devoted to bending, buckling and free vibration problems with special attention paid to the shear effects.

The subject of the study is a beam with unsymmetrically varying mechanical properties. A nonlinear hypothesis of deformation of the plane cross section of the beam is developed. Particular attention is paid to location of the neutral axis with consideration of the shear effect. Variability of the elastic modulus - Young's modulus in the depth direction of the beam is shown in Fig. 1.

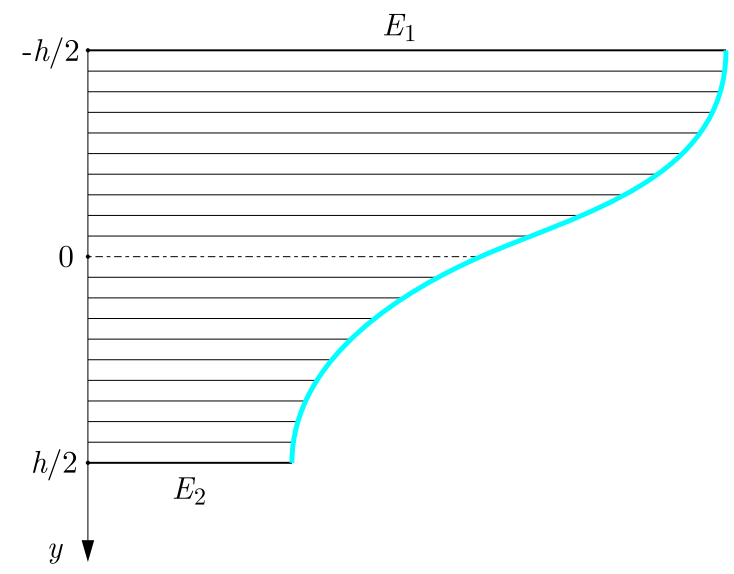

Fig. 1. Scheme of the elastic modulus variability in the depth direction of the beam

The values of elasticity moduli and mass density of the beam vary as follows

$$
\begin{aligned}
& \left.E(y)=\frac{1}{2} E_{1} 1+e_{2}-\left(1-e_{2}\right) \sin (\pi \eta)\right] \quad G(y)=\frac{1}{2} G_{1}\left[1+g_{2}-\left(1-g_{2}\right) \sin (\pi \eta)\right] \\
& \rho(y)=\frac{1}{2} \rho_{1}\left[1+\widetilde{\rho}_{2}-\left(1-\widetilde{\rho}_{2}\right) \sin (\pi \eta)\right]
\end{aligned}
$$

where: $e_{2}=E_{2} / E_{1}, g_{2}=G_{2} / G_{1}=\left(1+\nu_{1}\right) /\left(1+\nu_{2}\right) e_{2}, \widetilde{\rho}_{2}=\rho_{2} / \rho_{1}$ are dimensionless relative parameters, $E_{1}, E_{2}$ - Young's moduli, $\nu_{1}, \nu_{2}$ - Poisson's ratios, $\rho_{1}, \rho_{2}-$ mass densities, $\eta=y / h$ - dimensionless coordinate $(-0.5 \leqslant \eta \leqslant 0.5), h$ - depth of the beam.

The relationship between the relative density and Young's moduli ratio $\widetilde{\rho}_{2}=\sqrt{e_{2}}$ is assumed based on the papers by Chen et al. (2015, 2016b).

\section{Analytical model of the beam}

The nonlinear hypothesis is assumed for the purpose of modelling of the beam. A plane cross section before bending is no longer plane after bending of the beam (Fig. 2). This hypothesis is a generalization of the shear deformation theory for functionally graded structures.

Two coordinate systems are adopted $-x, y$ and $x_{1}, y_{1}$ (Fig. 2). The $x_{1}$ axis is the neutral axis, therefore, the displacement $v\left(x_{1}, t\right)$ is equivalent with $v(x, t)$. The coordinate $y_{1}=h\left(\eta+\eta_{0}\right)$, 


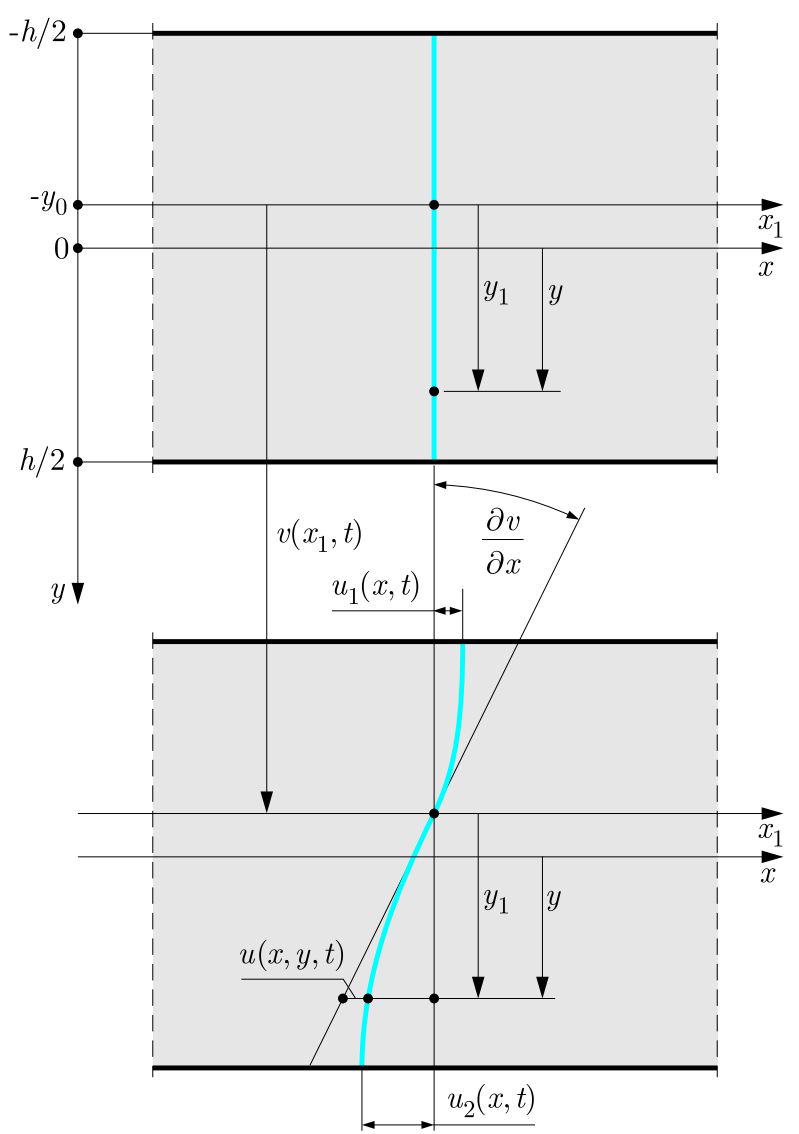

Fig. 2. Deformation of the plane cross section of the beam - the nonlinear hypothesis

where $\eta_{0}=y_{0} / h$, therefore, based on the above hypothesis, the displacement is in the following form

$$
u(x, y, t)=-h\left\{\left(\eta+\eta_{0}\right) \frac{\partial v}{\partial x}-\left[\sin (\pi \eta)+\sin \left(\pi \eta_{0}\right)\right] \psi(x, t)\right\}
$$

where: $v(x, t)$ - deflection, $\psi(x, t)$ - dimensionless function of the shear effect.

The shear effect displacements of upper and lower surfaces of the beam are as follows

$$
u_{1}(x, t)=-h\left[1-\sin \left(\pi \eta_{0}\right)\right] \psi(x, t) \quad u_{2}(x, t)=h\left[1+\sin \left(\pi \eta_{0}\right)\right] \psi(x, t)
$$

Then, the longitudinal strain

$$
\varepsilon_{x}(x, y, t)=\frac{\partial u}{\partial x}=-h\left\{\left(\eta+\eta_{0}\right) \frac{\partial^{2} v}{\partial x^{2}}-\left[\sin (\pi \eta)+\sin \left(\pi \eta_{0}\right)\right] \frac{\partial \psi}{\partial x}\right\}
$$

and the shear strain

$$
\gamma_{x y}(x, y, t)=\frac{\partial u}{\partial y}+\frac{\partial v}{\partial x}=\pi \cos (\pi \eta) \psi(x, t)
$$

The stresses - Hooke's law

$$
\sigma_{x}(x, y, t)=E(y) \varepsilon_{x}(x, y, t) \quad \tau_{x y}(x, y, t)=G(y) \gamma_{x y}(x, y, t)
$$

The simply supported beam with unsymmetrically varying mechanical properties of length $L$, depth $h$ and width $b$ is subjected to a uniformly distributed transverse load of intensity $q$ or to axial compression force $F_{0}$ (Fig. 3). 


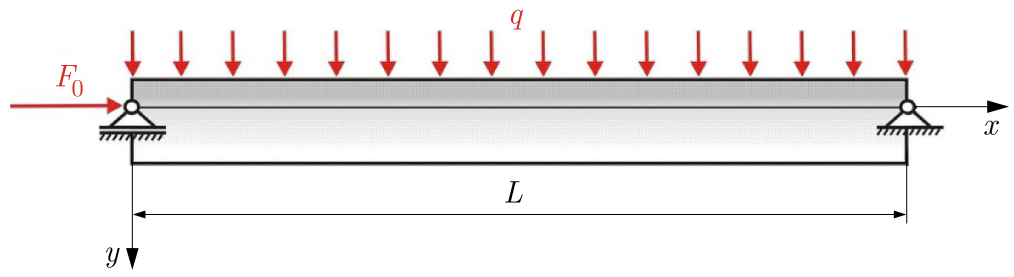

Fig. 3. Scheme of the beam and loads

The Hamilton principle

$$
\int_{t_{1}}^{t_{2}}\left[T-\left(U_{\varepsilon}-W\right)\right] d t=0
$$

where: $T$ is the kinetic energy, $U_{\varepsilon}$ - elastic strain energy, $W$ - work of the load

$$
\begin{aligned}
& T=\frac{1}{2} b h \rho_{b} \int_{0}^{L}\left(\frac{\partial v}{\partial t}\right)^{2} d x \quad U_{\varepsilon}=\frac{1}{2} b \int_{0}^{L} \int_{-h / 2}^{h / 2}\left[E(y) \varepsilon_{x}^{2}+G(y) \gamma_{x y}^{2}\right] d x d y \\
& W=\int_{0}^{L}\left[q v(x)+\frac{1}{2} F_{0}\left(\frac{\partial v}{\partial x}\right)^{2}\right] d x
\end{aligned}
$$

and the equivalent - mean mass density of the beam

$$
\rho_{b}=\frac{1}{h} \int_{-h / 2}^{h / 2} \rho(y) d y=\frac{1}{2}\left(\rho_{1}+\rho_{2}\right)=\frac{1}{2} \rho_{1}\left(1+\sqrt{e_{2}}\right)
$$

Substitution of expressions $(1.1)_{1}$ and $(1.1)_{2}$ for the elasticity moduli and expressions (2.3) and (2.4) for strains into expression $(2.7)_{2}$, after integration along depth of the beam, gives elastic strain energy as a functional of the two unknown functions

$$
U_{\varepsilon}=\frac{1}{4} E_{1} b h^{3} \int_{0}^{L}\left[C_{v v}\left(\frac{\partial^{2} v}{\partial x^{2}}\right)^{2}-2 C_{v \psi} \frac{\partial^{2} v}{\partial x^{2}} \frac{\partial \psi}{\partial x}+C_{\psi \psi}\left(\frac{\partial \psi}{\partial x}\right)^{2}+C_{\psi 0} \frac{\psi^{2}(x, t)}{h^{2}}\right] d x
$$

where

$$
\begin{aligned}
& C_{v v}=\frac{1}{12}\left[1-\frac{48}{\pi^{2}} \eta_{0}+12 \eta_{0}^{2}+\left(1+\frac{48}{\pi^{2}} \eta_{0}+12 \eta_{0}^{2}\right) e_{2}\right] \quad C_{\psi 0}=\frac{\pi^{2}}{4\left(1+\nu_{1}\right)}\left(1+g_{2}\right) \\
& C_{v \psi}=\frac{1}{2 \pi^{2}}\left\{\left(4-\pi^{2} \eta_{0}\right)\left[1-\sin \left(\pi \eta_{0}\right)\right]+\left(4+\pi^{2} \eta_{0}\right)\left[1+\sin \left(\pi \eta_{0}\right)\right] e_{2}\right\} \\
& C_{\psi \psi}=\frac{1}{2}-\sin \left(\pi \eta_{0}\right)+\sin ^{2}\left(\pi \eta_{0}\right)+\left[\frac{1}{2}+\sin \left(\pi \eta_{0}\right)+\sin ^{2}\left(\pi \eta_{0}\right)\right] e_{2}
\end{aligned}
$$

Based on Hamilton's principle (2.6) with consideration of expressions $(2.7)_{1},(2.7)_{3}$ and (2.9), two differential equations of motion are obtained in the following form

$$
\begin{aligned}
& b h \rho_{b} \frac{\partial^{2} v}{\partial t^{2}}+\frac{1}{2} E_{1} b h^{3}\left(C_{v v} \frac{\partial^{4} v}{\partial x^{4}}-C_{v \psi} \frac{\partial^{3} \psi}{\partial x^{3}}\right)+F_{0} \frac{\partial^{2} v}{\partial x^{2}}=q \\
& C_{v \psi} \frac{\partial^{3} v}{\partial x^{3}}-C_{\psi \psi} \frac{\partial^{2} \psi}{\partial x^{2}}+C_{\psi 0} \frac{\psi(x, t)}{h^{2}}=0
\end{aligned}
$$


The bending moment

$$
M_{b}(x)=b \int_{-h / 2}^{h / 2} y \sigma_{x}(x, y) d y
$$

Substituting expression (2.5) for the normal stress, after integration along depth of the beam, one obtains the following equation

$$
C_{v v} \frac{d^{2} v}{d x^{2}}-C_{v \psi} \frac{d \psi}{d x}=-2 \frac{M_{b}(x)}{E_{1} b h^{3}}
$$

It may be noticed that for static problems this equation is equivalent to equation $(2.10)_{1}$.

The position of the neutral axis is determined on the basis of the following condition - total axial force at the cross section

$$
\int_{-h / 2}^{h / 2} \sigma_{x}(x, y) d y=0
$$

Substituting expression (2.5) for the normal stress, after integration along depth of the beam, one obtains the following equation

$$
C_{N v} \frac{d^{2} v}{d x^{2}}-C_{N \psi} \frac{d \psi}{d x}=0
$$

where

$$
C_{N v}=\left(1+e_{2}\right) \eta_{0}-\frac{2}{\pi^{2}}\left(1-e_{2}\right) \quad C_{N \psi}=\frac{1}{2}\left(1-e_{2}\right)-\left(1+e_{2}\right) \sin \left(\pi \eta_{0}\right)
$$

Based on this condition, the position of the neutral axis $\eta_{0}=y_{0} / h$ is obtained (Fig. 2).

\section{Analytical solution of two differential equations of motion of the beam}

The system of two differential equations (2.10) for the beam is approximately solved with the use of two assumed functions

$$
v(x, t)=v_{a}(t) \sin \left(\pi \frac{x}{L}\right) \quad \psi(x, t)=\psi_{a}(t) \cos \left(\pi \frac{x}{L}\right)
$$

where: $v_{a}(t), \psi_{a}(t)$ are functions of time $t$, which in the case of static problems become parameters. These functions satisfy the conditions of a simply supported beam.

Substitution of functions (3.1) into equations (2.10) gives the following equations

$$
\begin{aligned}
& \left\{b h \rho_{b} \frac{d^{2} v_{a}}{d t^{2}}+\frac{1}{2}\left(\frac{\pi}{L}\right)^{4} E_{1} b h^{3}\left[C_{v v} v_{a}(t)-\frac{L}{\pi} C_{v \psi} \psi_{a}(t)\right]-\left(\frac{\pi}{L}\right)^{2} F_{0} v_{a}(t)\right\} \sin \left(\pi \frac{x}{L}\right)=q \\
& \left(\frac{\pi}{L}\right)^{3} C_{v \psi} v_{a}(t)-\left(\frac{\pi}{L}\right)^{2}\left[C_{\psi \psi}+\left(\frac{\lambda}{\pi}\right)^{2} C_{\psi 0}\right] \psi_{a}(t)=0
\end{aligned}
$$

where $\lambda=L / h$ is relative length of the beam.

From equation $(3.2)_{2}$, the function of time related to the shear effect is

$$
\psi_{a}(t)=\frac{\pi}{L} k_{s e} v_{a}(t)
$$


where the dimensionless coefficient of the shear effect is

$$
k_{s e}=\frac{C_{v \psi}}{C_{\psi \psi}+\left(\frac{\lambda}{\pi}\right)^{2} C_{\psi 0}}
$$

It may be noticed that the value of this coefficient decreases with increasing relative length of the beam.

Equation (3.2) 1 with consideration of expression (3.3) is in the following form

$$
\left[b h \rho_{b} \frac{d^{2} v_{a}}{d t^{2}}+\frac{1}{2}\left(\frac{\pi}{L}\right)^{4} E_{1} b h^{3}\left(C_{v v}-k_{s e} C_{v \psi}\right) v_{a}(t)-\left(\frac{\pi}{L}\right)^{2} F_{0} v_{a}(t)\right] \sin \left(\pi \frac{x}{L}\right)=q
$$

and after application of Galerkin's method is as follows

$$
b h \rho_{b} \frac{d^{2} v_{a}}{d t^{2}}+\frac{1}{2}\left(\frac{\pi}{L}\right)^{4} E_{1} b h^{3}\left(C_{v v}-k_{s e} C_{v \psi}\right) v_{a}(t)-\left(\frac{\pi}{L}\right)^{2} F_{0} v_{a}(t)=\frac{4}{\pi} q
$$

This equation is the base for detailed studies of the bending, buckling and free vibration of the simply supported beam with unsymmetrically varying mechanical properties.

Condition (2.14) for calculation of the position of the neutral axis of the beam (Fig. 2) with consideration of functions (3.1) and (3.2) 1 and expression (3.3) takes form of a transcendental equation

$$
\eta_{0}-k_{s e} \sin \left(\pi \eta_{0}\right)-\left(\frac{2}{\pi^{2}}-\frac{1}{2} k_{s e}\right) \frac{1-e_{2}}{1+e_{2}}=0
$$

It may be noticed that for large relative length of the beam $\left(\lambda \rightarrow \infty, k_{s e}=0\right)$, the position of the neutral axis is determined as

$$
\eta_{0}^{(l i m)}=\eta_{0}^{k_{s e}=0}=\frac{2}{\pi^{2}} \frac{1-e_{2}}{1+e_{2}}
$$

Example. The following data of the beam are assumed: Poisson's ratios: $\nu_{1}=\nu_{2}=0.33$, $e_{2}=0.010,0.025,0.050$, and relative length $\lambda=4,6, \ldots, 14, \infty$. The dimensionless values $\eta_{0}(3.7)$ and $\eta_{0}^{(\mathrm{lim})}$ (3.8) of the position of the neutral axis of the beam are specified in Table 1.

Table 1. The dimensionless values $\eta_{0}$ of the position of the neutral axis

\begin{tabular}{|c|c|c|c|c|c|c|c|}
\hline \multirow{2}{*}{$e_{2}$} & \multicolumn{7}{|c|}{$\lambda$} \\
\cline { 2 - 8 } & 4 & 6 & 8 & 10 & 12 & 14 & $\infty$ \\
\hline \hline 0.010 & 0.2019 & 0.2001 & 0.1995 & 0.1992 & 0.1990 & 0.1989 & 0.1986 \\
\hline 0.025 & 0.1962 & 0.1943 & 0.1936 & 0.1933 & 0.1931 & 0.1930 & 0.1928 \\
\hline 0.050 & 0.1870 & 0.1850 & 0.1843 & 0.1839 & 0.1838 & 0.1836 & 0.1833 \\
\hline
\end{tabular}

The graph of the dimensionless values $\eta_{0}$ and $\eta_{0}^{(l i m)}$ of the position of the neutral axis of the beam is shown in Fig. 4.

For the homogeneous beam $\left(e_{2}=1\right)$, the neutral axis is located in the middle depth of the beam $\left(\eta_{0}=0\right)$. 


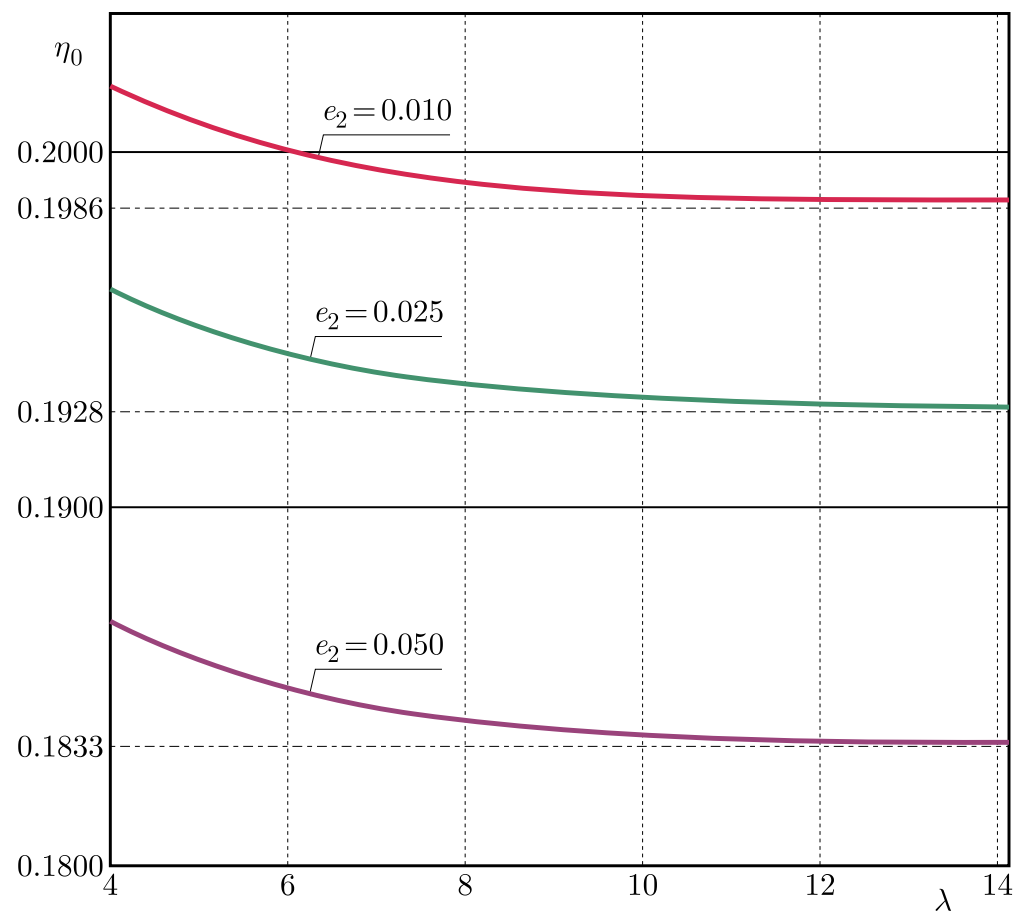

Fig. 4. The graph of dimensionless values $\eta_{0}$ for the position of the neutral axis of the beam

\section{Bending of the beam, static problem - analytical solution}

The simply supported beam with unsymmetrically varying mechanical properties is subjected to a uniformly distributed transverse load of intensity $q$ (Fig. 3). On the basis of equation (3.6) for the static problem $\left(d^{2} v_{a} / d t^{2}=0\right)$ and $F_{0}=0$, the relative maximum deflection is obtained

$$
\widetilde{v}_{\max }=\frac{v_{\max }}{L}=k_{v \max } \frac{q \lambda^{3}}{E_{1} b}
$$

where the dimensionless coefficient of the maximal deflection is

$$
k_{v \max }=\frac{8}{\pi^{5}\left(C_{v v}+k_{s e} C_{v \psi}\right)}
$$

In the case of large relative length of the beam $\left(\lambda \rightarrow \infty, k_{s e}=0\right)$, this coefficient of the maximun deflection is

$$
k_{v \max }^{(\lim )}=\frac{8}{\pi^{5} C_{v v}}
$$

Example. The following data of the beam are assumed: Poisson's ratios: $\nu_{1}=\nu_{2}=0.33$, $e_{2}=0.010,0.050, \ldots, 0.50,1.0$, and relative length $\lambda=5,10,15,20, \infty$. The values of the dimensionless coefficient of the maximum deflection $k_{v \max }$ and $k_{v \max }^{(\text {lim) }}$ are specified in Table 2 .

The graph of the values of the dimensionless coefficient of the maximum deflection $k_{v \max }$ and $k_{v \max }^{(\text {lim })}$ is shown in Fig. 5.

For the homogeneous beam $\left(e_{2}=1\right)$ of large relative length, the dimensionless coefficient of the maximum deflection $k_{v \max }^{(l i m)}=48 / \pi^{5}$. 
Table 2. The dimensionless coefficient $k_{v \max }$ of the maximum deflection

\begin{tabular}{|c|c|c|c|c|c|}
\hline \multirow{2}{*}{$e_{2}$} & \multicolumn{5}{|c|}{$\lambda$} \\
\cline { 2 - 6 } & 5 & 10 & 15 & 20 & $\infty$ \\
\hline \hline 0.01 & 0.6215 & 0.5978 & 0.5934 & 0.5919 & 0.5899 \\
\hline 0.05 & 0.5314 & 0.5084 & 0.5042 & 0.5027 & 0.5008 \\
\hline 0.10 & 0.4550 & 0.4329 & 0.4288 & 0.4274 & 0.4256 \\
\hline 0.25 & 0.3312 & 0.3116 & 0.3080 & 0.3067 & 0.3051 \\
\hline 0.50 & 0.2431 & 0.2267 & 0.2237 & 0.2226 & 0.2213 \\
\hline 1.0 & 0.1733 & 0.1610 & 0.1587 & 0.1579 & 0.1569 \\
\hline
\end{tabular}

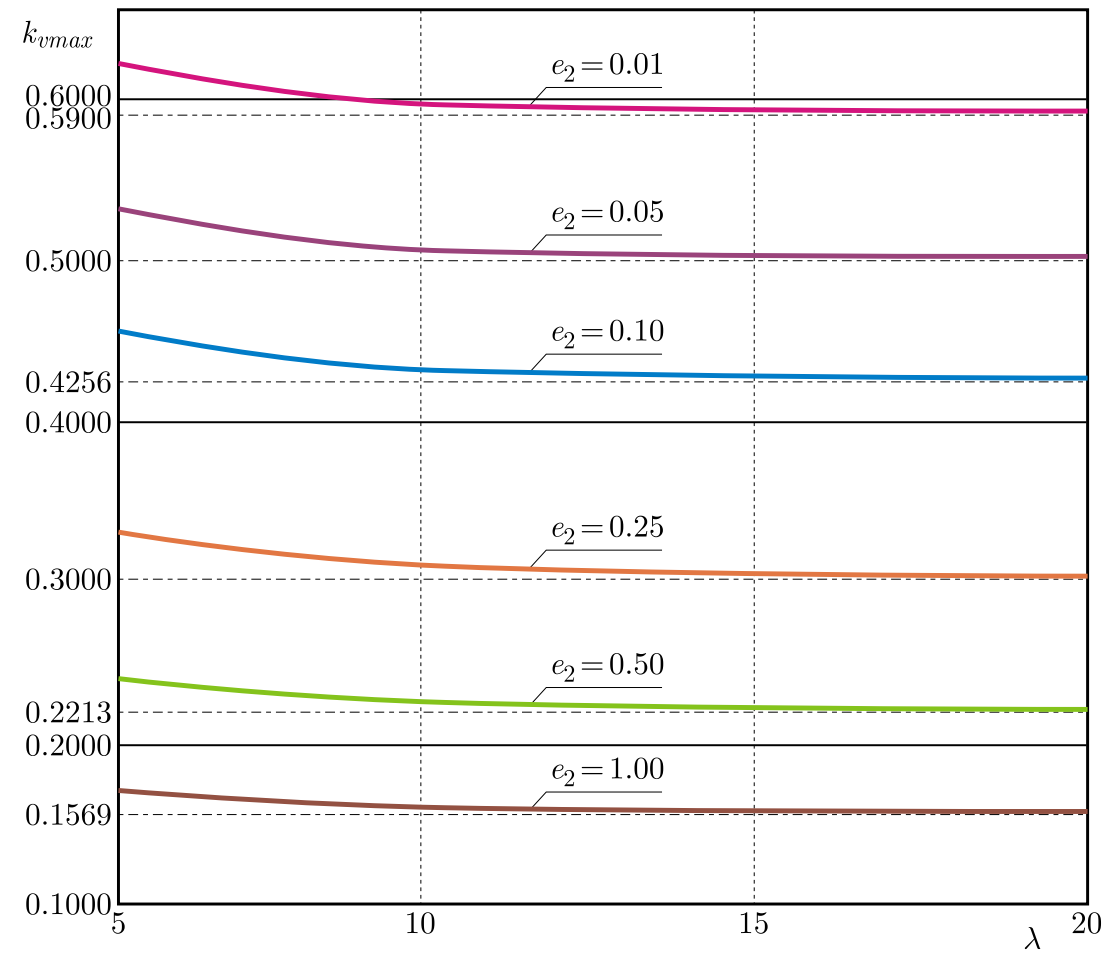

Fig. 5. The graph of the values of the dimensionless coefficient of the maximum deflection

\section{Buckling of the beam, static problem - analytical solution}

The simply supported beam with unsymmetrically varying mechanical properties is subjected to axial compression with the force $F_{0}$ (Fig. 3). On the basis of equation (3.6) for static problem $\left(d^{2} v_{a} / d t^{2}=0\right)$ and $q=0$, the critical force is obtained

$$
F_{0, C R}=\left(\frac{\pi}{\lambda}\right)^{2} k_{F C R} E_{1} b h
$$

where the dimensionless coefficient of the critical force is

$$
k_{F C R}=\frac{1}{2}\left(C_{v v}-k_{s e} C_{v \psi}\right)
$$

In the case of large relative length of the beam $\left(\lambda \rightarrow \infty, k_{s e}=0\right)$, this coefficient of the critical force is

$$
k_{F C R}^{(l i m)}=\frac{1}{2} C_{v v}
$$


Example. The following data of the beam are assumed: Poisson's ratios: $\nu_{1}=\nu_{2}=0.33$, $e_{2}=0.010,0.050, \ldots, 0.50,1.0$, and relative length $\lambda=25,30,35,40, \infty$. The values of the dimensionless coefficient of the critical force $k_{F C R}$ and $k_{F C R}^{(\text {lim })}$ are specified in Table 3 . The $\lambda$ values in the buckling problem are larger than those for bending, since the critical loads for short beams would be very high and, therefore, elastic-plastic buckling would arise.

Table 3. The values of the dimensionless coefficient $k_{F C R}$ and $k_{F C R}^{(l i m)}$ of the critical force

\begin{tabular}{|c|c|c|c|c|c|}
\hline \multirow{2}{*}{$e_{2}$} & \multicolumn{5}{|c|}{$\lambda$} \\
\cline { 2 - 6 } & 25 & 30 & 35 & 40 & $\infty$ \\
\hline \hline 0.01 & 0.022112 & 0.022126 & 0.022135 & 0.022141 & 0.022159 \\
\hline 0.05 & 0.026038 & 0.026058 & 0.026070 & 0.026077 & 0.026102 \\
\hline 0.10 & 0.030629 & 0.030655 & 0.030671 & 0.030681 & 0.030714 \\
\hline 0.25 & 0.042698 & 0.042742 & 0.042769 & 0.042787 & 0.042844 \\
\hline 0.50 & 0.058845 & 0.058916 & 0.058959 & 0.058987 & 0.059078 \\
\hline 1.0 & 0.082985 & 0.083091 & 0.083155 & 0.083197 & 0.083333 \\
\hline
\end{tabular}

The graph of the values of the dimensionless coefficient of the critical force $k_{F C R}$ and $k_{F C R}^{(l i m)}$ is shown in Fig. 6.

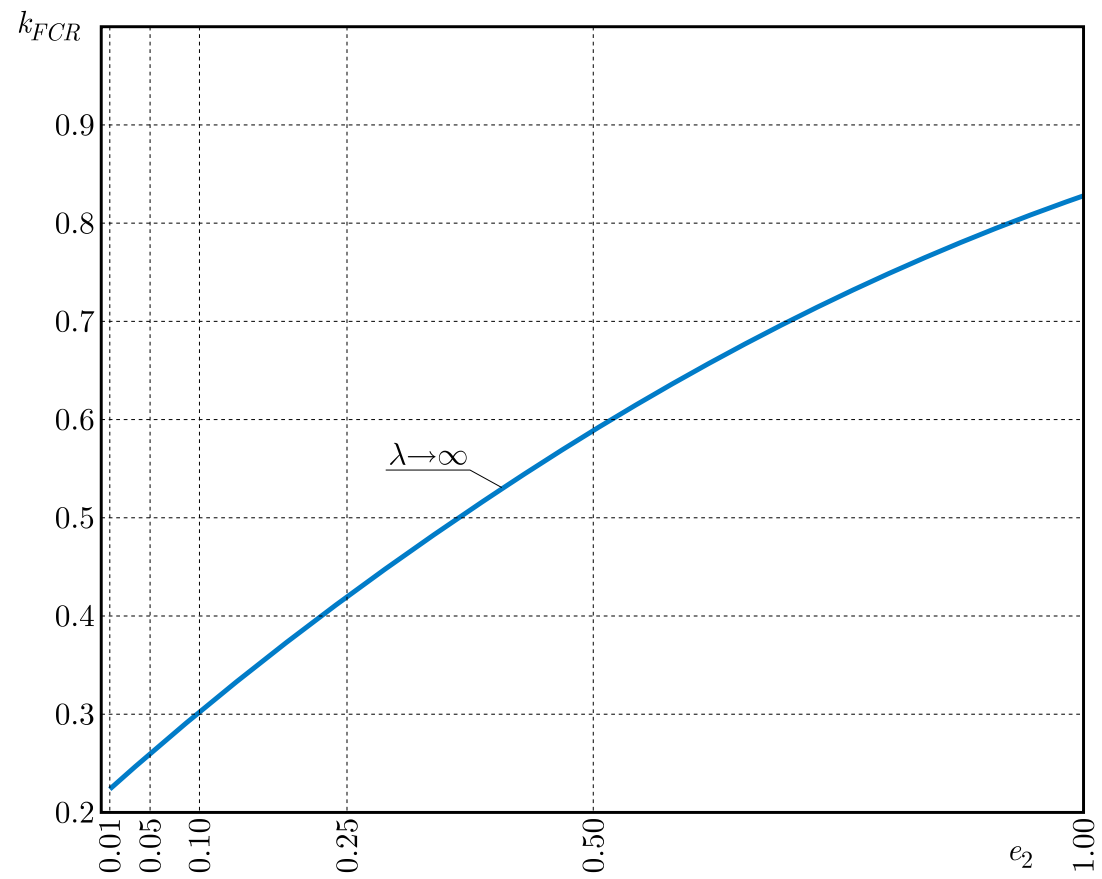

Fig. 6. The graph of the values of the dimensionless coefficient of the critical force

For the homogeneous beam $\left(e_{2}=1\right)$ of large relative length, the dimensionless coefficient of the critical force $k_{F C R}^{(l i m)}=1 / 12$.

\section{Free vibration of the beam, dynamic problem - analytical solution}

The simply supported beam with unsymmetrically varying mechanical properties is not loaded $\left(q=0, F_{0}=0\right)$ (Fig. 3). Equation (3.6) for the dynamic problem is as follows

$$
\rho_{b} \frac{d^{2} v_{a}}{d t^{2}}+\frac{1}{2}\left(\frac{\pi}{L}\right)^{4} E_{1} h^{2}\left(C_{v v}-k_{s e} C_{v \psi}\right) v_{a}(t)=0
$$


The equation is solved with the use of the assumed function

$$
v_{a}(t)=v_{a} \sin (\omega t)
$$

where: $v_{a}$ is the amplitude of flexural vibration, $\omega$ - fundamental natural frequency.

Substituting this function into equation (6.1), after simple transformation, one obtains the fundamental natural frequency

$$
\omega=\left(\frac{\pi}{\lambda}\right)^{2} k_{\omega} \sqrt{\frac{E_{1}}{\rho_{b} h^{2}}}
$$

where the dimensionless coefficient of the fundamental natural frequency is

$$
k_{\omega}=\sqrt{\frac{1}{2}\left(C_{v v}-k_{s e} C_{v \psi}\right)}=\sqrt{k_{F C R}}
$$

Taking into account expression (5.3), one formulates $k_{\omega}^{(\mathrm{lim})}=\sqrt{k_{F C R}^{(\mathrm{lim})}}$.

Example. The following data of the beam are assumed: Poisson's ratios: $\nu_{1}=\nu_{2}=0.33$, $e_{2}=0.010,0.050, \ldots, 0.80,1.0$, and relative length $\lambda=5,10,15,25, \infty$. The values of the dimensionless coefficient of the fundamental natural frequency $k_{\omega}$ and $k_{\omega}^{(\mathrm{lim})}$ are specified in Table 4.

Table 4. The values of the dimensionless coefficient $k_{\omega}$ and $k_{\omega}^{(\text {lim })}$ of the natural frequency

\begin{tabular}{|c|c|c|c|c|c|}
\hline \multirow{2}{*}{$e_{2}$} & \multicolumn{5}{|c|}{$\lambda$} \\
\cline { 2 - 6 } & 5 & 10 & 15 & 25 & $\infty$ \\
\hline \hline 0.01 & 0.14502 & 0.14787 & 0.14842 & 0.14870 & 0.14886 \\
\hline 0.05 & 0.15683 & 0.16034 & 0.16101 & 0.16136 & 0.16156 \\
\hline 0.10 & 0.16949 & 0.17376 & 0.17458 & 0.17501 & 0.17526 \\
\hline 0.25 & 0.19866 & 0.20481 & 0.20601 & 0.20663 & 0.20699 \\
\hline 0.50 & 0.23187 & 0.24011 & 0.24173 & 0.24258 & 0.24306 \\
\hline 0.80 & 0.25984 & 0.26954 & 0.27146 & 0.27246 & 0.27303 \\
\hline 1.0 & 0.27465 & 0.28497 & 0.28701 & 0.28807 & 0.28868 \\
\hline
\end{tabular}

The graph of the values of the dimensionless coefficient of the fundamental natural frequency $k_{\omega}$ and $k_{\omega}^{(l i m)}$ is shown in Fig. 7 .

In the case of the homogeneous beam $\left(e_{2}=1\right)$ of large relative length, the dimensionless coefficient of the critical force $k_{F C R}^{(\text {lim })}=\sqrt{3} / 6$.

\section{Numerical calculations - FEM study}

\subsection{Numerical FEM model}

The numerical analysis of the beam with unsymmetrically varying mechanical properties is carried out with the help of the SolidWorks software. The simulation assumed the same geometry parameters and mechanical properties as those used in the analytical calculations.

The beam is modelled using 3D finite elements in 20 layers, each with different mechanical properties satisfying expressions (1.1). Taking into account symmetry of the structure, a half of the beam is considered (Fig. 8). 


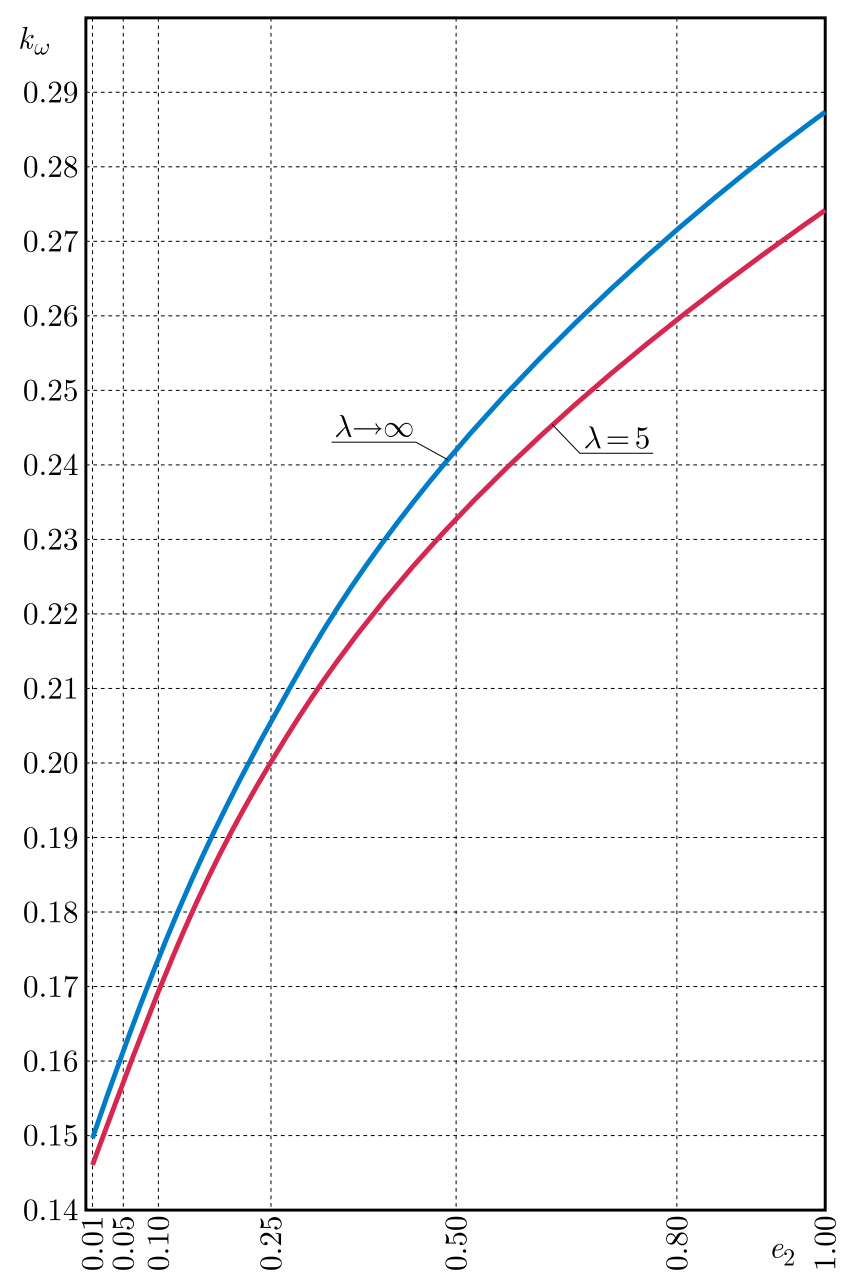

Fig. 7. The graph of the values of the dimensionless coefficient of the fundamental natural frequency

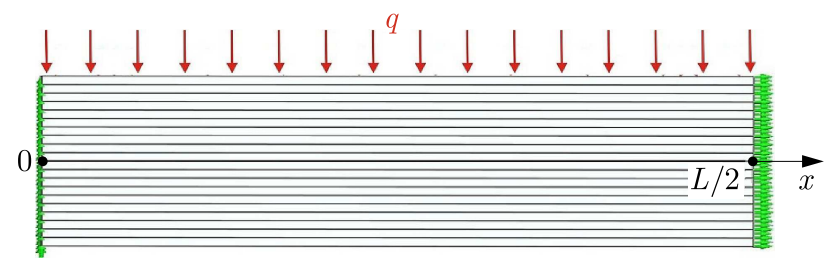

Fig. 8. Boundary conditions and load for the bending problem in the FEM study

Therefore, the following boundary conditions are adopted:

- for $x=0$ - the simple support $-v(0)$ displacements in the $y$ direction are zero;

- for $x=L / 2$ - the middle of the beam $-u(L / 2)$ displacements in the $x$ direction are zero.

The numerical study of bending, buckling and free vibration is restrained to the $x y$-plane, similarly as in the case of the analytical approach.

SolidWorks calculations have been carried out for beams with a rectangular cross-section of depth $h=80 \mathrm{~mm}$, width $b=20 \mathrm{~mm}$, and length values $L=\lambda h(400 \mathrm{~mm} \leqslant L \leqslant 3200 \mathrm{~mm})$.

\subsection{Bending of the beam, static problem - numerical FEM solution}

The beam is subjected to a uniformly distributed load of intensity $q$. A view to the bent half-beam is shown in Fig. 9. 


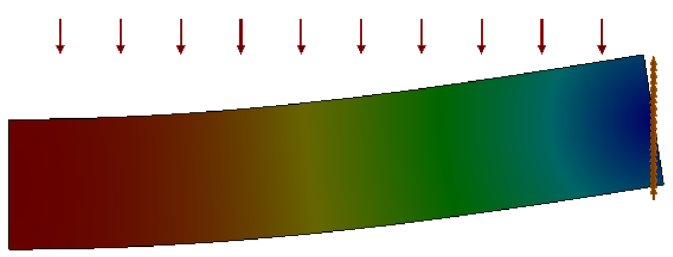

Fig. 9. Deflection of the beam (SolidWorks simulation)

Results of the study are maximum deflections $v_{\max }[\mathrm{mm}]$. Based on expressions (4.1) and (4.2), values of the dimensionless coefficient $k_{v \max }$ are calculated. These values are specified in Table 5.

Table 5. The values of the dimensionless coefficient $k_{v \max }$ of the maximum deflection (FEM study)

\begin{tabular}{|c|c|c|c|c|}
\hline \multirow{2}{*}{$e_{2}$} & \multicolumn{4}{|c|}{$\lambda$} \\
\cline { 2 - 5 } & 5 & 10 & 15 & 20 \\
\hline \hline 0.01 & 0.6136 & 0.5950 & 0.5886 & 0.5875 \\
\hline 0.05 & 0.5248 & 0.5050 & 0.5007 & 0.5000 \\
\hline 0.10 & 0.4496 & 0.4295 & 0.4257 & 0.4250 \\
\hline 0.25 & 0.3272 & 0.3090 & 0.3062 & 0.3050 \\
\hline 0.50 & 0.2400 & 0.2250 & 0.2222 & 0.2216 \\
\hline 1.0 & 0.1696 & 0.1595 & 0.1580 & 0.1569 \\
\hline
\end{tabular}

Values of the relative difference between analytical and FEM solutions are below $2.2 \%$. The highest difference occurs for small relative length values $\lambda$. For greater $\lambda$ values, the difference decreases.

\subsection{Buckling of the beam, static problem - numerical FEM solution}

The half-beam under compression is shown in Fig. 10. Cross-sections and variation of mechanical properties of the beam are the same as in the case of bending. Therefore, the buckling is analysed only in the $x y$-plane, similarly as for bending and free vibration. In order to avoid lateral buckling the $z$-displacements in the whole $x y$-plane are zeroed.

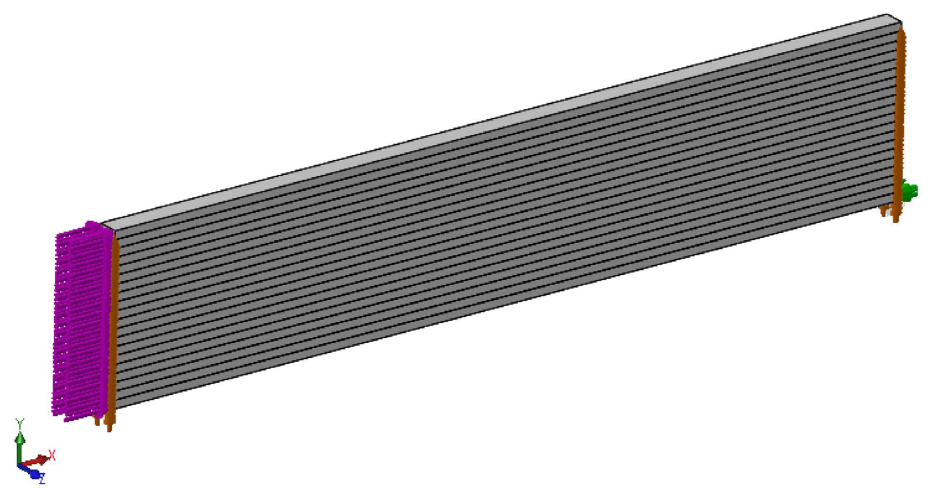

Fig. 10. Boundary conditions and load for the buckling problem in the FEM study

Buckling shape of the beam resulting from the numerical study is shown in Fig. 11.

Results of the study are critical force values $F_{0, C R}[\mathrm{~N}]$. Based on expressions (5.1) and (5.2), values of the dimensionless coefficient $k_{F C R}$ are calculated. These values are specified in Table 6 .

Values of the relative difference between analytical and FEM solutions are below $4.6 \%$. 


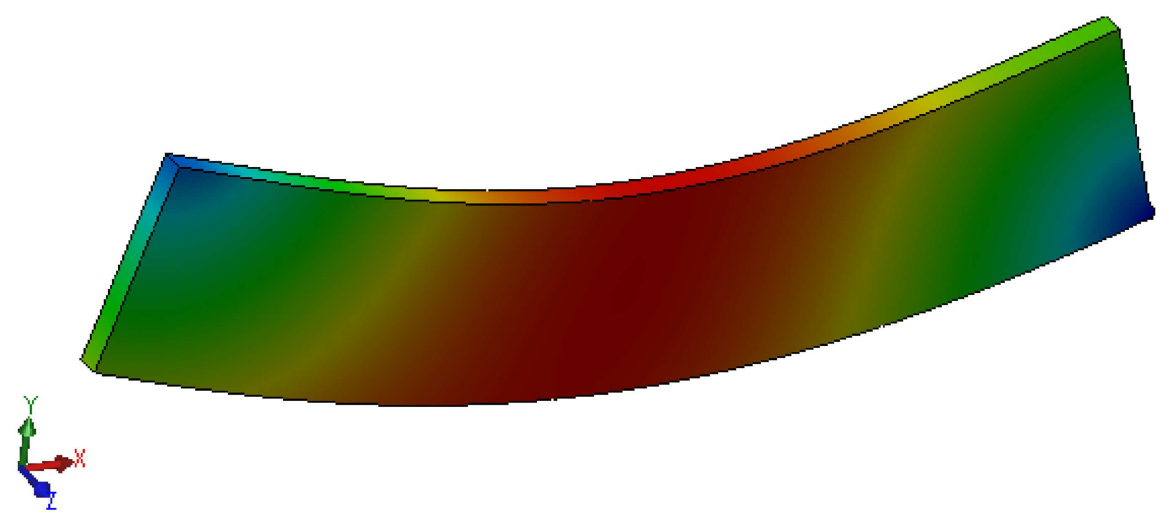

Fig. 11. Buckling shape of the beam (SolidWorks simulation)

Table 6. The values of the dimensionless coefficient $k_{F C R}$ of the critical force (FEM study)

\begin{tabular}{|c|c|c|c|c|}
\hline \multirow{2}{*}{$e_{2}$} & \multicolumn{4}{|c|}{$\lambda$} \\
\cline { 2 - 5 } & 25 & 30 & 35 & 40 \\
\hline \hline 0.01 & 0.0211 & 0.0213 & 0.0215 & 0.0216 \\
\hline 0.05 & 0.0252 & 0.0255 & 0.0256 & 0.0258 \\
\hline 0.10 & 0.0300 & 0.0302 & 0.0304 & 0.0306 \\
\hline 0.25 & 0.0424 & 0.0429 & 0.0431 & 0.0435 \\
\hline 0.50 & 0.0591 & 0.0598 & 0.0602 & 0.0607 \\
\hline 1.0 & 0.0806 & 0.0811 & 0.0815 & 0.0817 \\
\hline
\end{tabular}

\subsection{Free vibration of the beam, dynamic problem - numerical FEM solution}

Free vibrations are computed with the SolidWorks software for the FEM model composed of 10 layers of varying mechanical properties. A half-beam is adopted with the boundary conditions shown in Fig. 12.

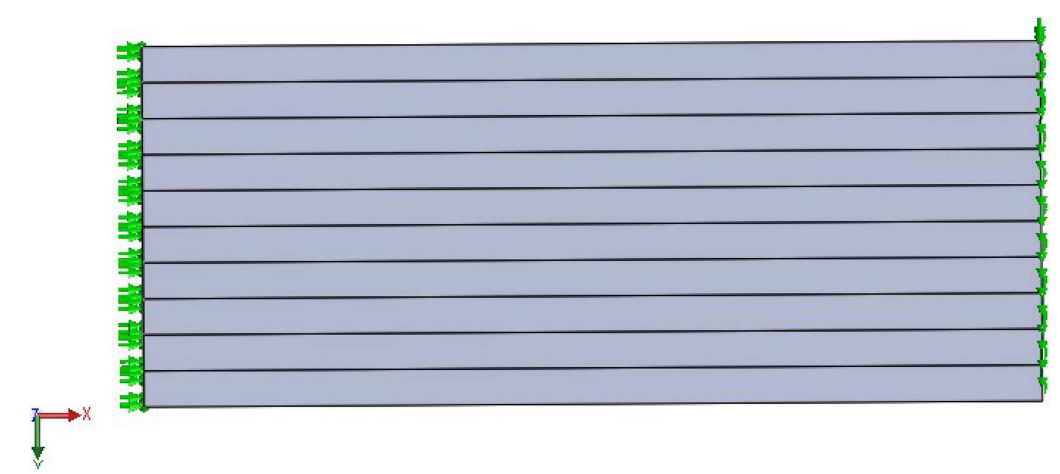

Fig. 12. Half-beam model used in the free-vibration calculation

The middle of the beam is placed in the left-hand side of the illustration. Hence, the $x$ and $z$ displacements are zeroed there. The right-hand part of the beam is simply supported and, therefore, the displacement $y$ is blocked.

The SolidWorks simulation tool used to compute the free-vibration frequencies provides angular frequencies $\omega$ of particular vibration modes. Nevertheless, in order to compare the analytical and numerical results, the dimensionless coefficient of the natural frequencies should be calculated for each case specified in Table 4. Taking into account expressions (2.8) and (6.3), one obtains 


$$
k_{\omega}=\omega h\left(\frac{\lambda}{\pi}\right)^{2} \sqrt{\frac{\rho_{1}\left(1+\sqrt{e_{2}}\right)}{2 E_{1}}}
$$

In the case of the example presented in Fig. 13, the following data are assumed: $e_{2}=0.01$, $b=20 \mathrm{~mm}, h=80 \mathrm{~mm}, L=400 \mathrm{~mm}$. Such a data set corresponds to the upper row and left-hand column of Table 4 . The angular frequency in this case is equal to $\omega=5093 \mathrm{rad} / \mathrm{s}$, which gives $k_{\omega}=0.14240$.

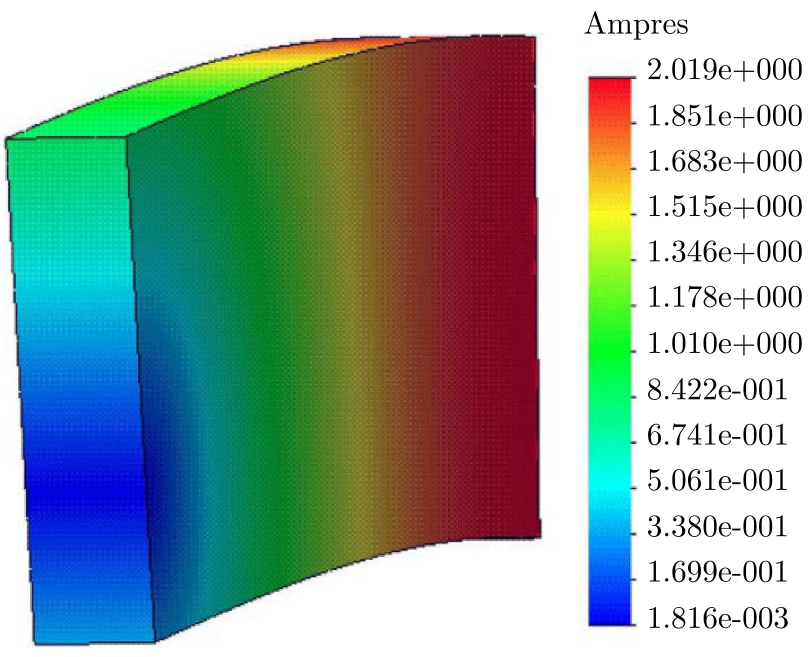

Fig. 13. An example result for $e_{2}=0.01$ and $\lambda=5$

The results $k_{\omega}$ of all the considered cases are presented in Table 7 .

Table 7. Values of the dimensionless coefficient $k_{\omega}$ of the natural frequency computed numerically

\begin{tabular}{|c|c|c|c|c|}
\hline \multirow{2}{*}{$e_{2}$} & \multicolumn{4}{|c|}{$\lambda$} \\
\cline { 2 - 5 } & 5 & 10 & 15 & 25 \\
\hline \hline 0.01 & 0.14240 & 0.14609 & 0.14680 & 0.14714 \\
\hline 0.05 & 0.15530 & 0.15987 & 0.16075 & 0.16119 \\
\hline 0.10 & 0.16775 & 0.17326 & 0.17434 & 0.17489 \\
\hline 0.25 & 0.19658 & 0.20426 & 0.20578 & 0.20655 \\
\hline 0.50 & 0.22962 & 0.23955 & 0.24153 & 0.24254 \\
\hline 0.80 & 0.25743 & 0.26893 & 0.27125 & 0.27243 \\
\hline 1.0 & 0.27214 & 0.28435 & 0.28681 & 0.28805 \\
\hline
\end{tabular}

They perfectly comply with the results of Table 4 obtained analytically. The relative difference values between analytical and FEM solutions are below $2 \%$. The highest difference, equal to $1.8 \%$, occurs for the example case mentioned above, whereas for the others they are significantly smaller.

\section{Conclusions}

The neutral axis of the studied beam deviates from the geometric centre of the rectangular cross section as a result of unsymmetrical properties of the material. The location of the neutral axis is affected by the shear effect (Fig. 4). This effect is meaningful in the case of short beams and disappears for longer ones. The values of deflection, critical load and free-vibration frequencies depend on the position of the neutral axis. Therefore, in the case of the analytical approach, the 
position of the axis should be determined first of all. The values of deflection, critical load and free-vibration frequencies obtained analytically have been compared to those computed with the SolidWorks software. It may be noticed that the difference between both sets of the results does not exceed $5 \%$.

\section{References}

1. Birman V., Byrd L.W., 2007, Modeling and analysis of functionally graded materials and structures, Applied Mechanics Reviews, 60, 195-216

2. Chen D., Kitipornchai S., Yang J., 2016a, Nonlinear free vibration of shear deformable sandwich beam with a functionally graded porous core, Thin-Walled Structures, 107, 39-48

3. Chen D., Yang J., Kitipornchai S., 2015, Elastic buckling, and static bending of shear deformable functionally graded porous beam, Composite Structures, 133, 54-61

4. Chen D., Yang J., Kitipornchai S., 2016b, Free and force vibrations of shear deformable functionally graded porous beams, International Journal of Mechanical Sciences, 108-109, 14-22

5. Debowski D., Magnucki K., Malinowski M., 2010, Dynamic stability of a metal foam rectangular plate, Steel and Composite Structures, 10, 2, 151-168

6. Feyzi M.R., Khorshidvand A.D., 2017, Axisymmetric post-buckling behaviour of saturated porous circular plates, Thin-Walled Structures, 112, 149-158

7. Jun L., Xiang H., Xiaobin L., 2016, Free vibration analyses of axially loaded laminated composite beams using a unified higher-order shear deformation theory and dynamic stiffness method, Composite Structures, 158, 308-322

8. Kapuria S., Bhattacharyya M., Kumar A.N., 2008, Bending and free vibration response of layered functionally graded beams: A theoretical model and its experimental validation, Composite Structures, 82, 390-402

9. Kolakowski Z., Mania R.J., 2015, Dynamic response of thin FG plates with a static unsymmetrical stable postbuckling path, Thin-Walled Structures, 86, 10-17

10. Kubiak T., 2005, Dynamic buckling of thin-walled composite plates with varying width-wise material properties, International Journal of Solids and Structures, 45, 5555-5567

11. KubiaK T., 2011, Estimation of dynamic buckling for composite columns with open cross-sections, Computers and Structures, 89, 21-22, 2001-2009

12. Li L., HU Y., Nonlinear bending and free vibration analyses of nonlocal strain gradient beams made of functionally graded material, International Journal of Mechanical Sciences, 107, 77-97

13. Magnucka-Blandzi E., 2011, Dynamic stability and static stress state of a sandwich beam with a metal foam core using three modified Timoshenko hypothesis, Mechanics of Advanced Materials and Structures, 18, 2, 147-158

14. Mahi A., Bedia E.A.A., Tounsi A., 2015, A new hyperbolic deformation theory for bending and free vibration analysis of isotropic, functionally graded, sandwich and laminated composite plates, Applied Mathematical Modelling, 39, 2489-2508

15. Mojahedin A., Jabbari M., Khorshidvand A., Eslami M., 2016, Buckling analysis of functionally graded circular plates made of saturated porous material based on higher order shear deformation theory, Thin-Walled Structures, 99, 83-90

16. Sayyad A.S., Ghugal Y.M., 2017, Bending, buckling and free vibration of laminated composite and sandwich beams: A critical review of literature, Composite Structures, 171, 486-504

17. Song M., Kitipornchai S., Yang J., 2017, Free and force vibrations of functionally graded polymer composite plates reinforced with graphene nanoplatelets, Composite Structures, 159, $579-588$ 
18. Smyczynski M.J., Magnucka-Blandzi E., 2018, Three-point bending of a sandwich beam with two binding layers - Comparison of two nonlinear hypotheses, Composite Structures, 183, 96-102

19. Thai H.-T., Vo T.P., 2012, Bending and free vibration of functionally graded beams using various higher-order shear deformation beam theories, International Journal of Mechanical Sciences, 62, 57-66

20. Thai H.-T., Vo T.P., 2013, A new sinusoidal shear deformation theory for bending, buckling, and vibration of functionally graded plates, Applied Mathematical Modelling, 37, 3269-3281

21. Zenkour A.M., 2006, Generalized shear deformation theory for bending analysis of functionally graded plates, Applied Mathematical Modelling, 30, 67-84

22. Zhang Y., Wang S., Loughlan J., 2006, Free vibration analysis of rectangular composite laminates using a layerwise cubic B-spline finite strip method, Thin-Walled Structures, 44, 601-622

Manuscript received September 21, 2017; accepted for print June 7, 2018 